\title{
Commission 42: Close Binaries
}

\author{
PRESIDENT: Alvaro Giménez \\ VICE-PRESIDENT: Slavek Rucinski \\ ORGANIZING COMMITTEE: P. Szkody, D. Gies, Y.-W. Kang,
}

J. Linsky, M. Livio, N. Morrell, R. Hilditch, B. Nordström, I. Ribas,

E. Sion, and S. Vrielman

\section{Introduction}

The triennial report from Commission 42 covers various topics like massive binaries, contact systems, cataclysmic variables and low-mass binary stars. We try in a number of sections to provide an update on the current status of the main research areas in the field of close binaries. It is not a formal review, even complete or comprehensive, but an attempt to bring the main topics on recent research to astronomers working in other fields. References are also not comprehensive and simply added to the text to help the reader looking for deeper information on the subject. For this reason, we have chosen to include references (sometimes incomplete for ongoing work) not in a list at the end but integrated with the main text body. Complete references and additional sources can be easily obtained through web access of ADS or SIMBAD. Furthermore, the summary of papers on close-binary research contained in the Bibliography of Close Binaries (BCB) can be accessed from the web site of Commission 42. I would like to express the gratitude of the commission for the careful work of Colin Scarfe as Editor-in-Chief of BCB and Andras Holl and Attila Sragli for maintaining the web pages of the Commission within the structure of Division V. Finally, K. Olah and J. Jurcsik are gratefully acknowledged for their continued support as editors of the Information Bulletin on Variable Stars (IBVS), also accessible through the commission web page.

The style of each section may be slightly different to each other. This reflects the actual wish of the main contributor and was kept at the time of integrating the report as a whole. Section 2 was mainly put together by B. Nordström, section 3 by D. Gies and N. Morrell, section 4 by S. Rucinski, section 5 by E. Sion and P. Szkody, section 6 by I. Ribas and the working groups reports by their corresponding chairperson (P. Szkody and I. Ribas, respectively). I would like to thank all members of Commission 42 for their work and cooperation in the IAU tasks, including the preparation of this report.

The area of research in stellar astrophysics devoted to the structure, formation and evolution of close binaries is still providing crucial new hints in modern astrophysics. Particularly important during the last years have been the understanding of accretion processes around compact stars, the study of massive binaries in other galaxies and very low-mass systems in our neighborhood.

The launch of Integral and Spitzer has opened new eyes to the world of close binaries from space while robotic telescopes and wide-field photometry have increased the efficiency of ground-based instruments in the form of large sky surveys. Furthermore, large telescopes are now in operation pushing the limits for the analysis of distant and faint objects. The beginning of the new millennium has brought a revival of the interest in close binaries and a routine phase of several years is being replaced by new exploratory projects with the use of new technologies to achieve challenging objectives. The coming General Assembly in Prague is a good example of this situation and the organizing 
committee is warmly thanked for the support to hold a Symposium specifically devoted to Binary Stars as Critical Tools and Tests in Contemporary Astrophysics.

\section{Conferences and Books}

During the past triennium, many meetings on close binaries were held and many more included topics related to close-binary research. The proceedings from those meetings serve as a written record on the work by the very active research community. The following list of meetings and their proceedings is by no means complete, but includes many of the important international meetings:

"Interacting Stars", Nordic-Baltic Research School, Moletai Observatory, Lithuania, August 2002; "New Directions for Close Binary Studies: The Royal Road to the Stars", Çanakkale Astrophysics Workshop, Turkey 2002 (Publ. by COMU Astrophysics Research Center); "The Environment and Evolution of Double and Multiple Stars", IAU Coll. 191, Merida, Mexico, February, 2003, (RevMexAA, Vol. 21, Eds. C. Allen \& C. Scarfe); "Compact Binaries in the Galaxy and Beyond", IAU Coll. 194, La Paz, Mexico 2003 (RevMexAA, Vol. 20, Eds. G. Tovmassian \& E.M. Sion); "Variable Stars in the Local Group", IAU Coll. 193, Christchurch, New Zealand 2003, (ASP Conf. Ser. 310, Eds. D.W. Kurtz \& K.R. Pollard); "Extra-galactic Binaries", IAU JD13, Sydney, Australia 2003 (New Astronomy Reviews, Vol. 48, issue 9, Eds. I. Ribas \& A. Giménez); "Spectroscopically and Spatially Resolving the Components of Close Binary Stars", Dubrovnik, Croatia 2003 (ASP Conf. Ser. 318, Eds. R. W. Hilditch, H. Hensberge \& K. Pavlovski); "Zdenek Kopal's Binary Star Legacy", Litomyle, Czech Republic 2004, (A\&SS, Ed. H. Drechsel); "Active stars and interacting binaries", 13th Joint European and National Astronomical Meeting, Budapest, Hungary 2003; "Third Granada Workshop on Stellar Structure: Tidal Evolution and Oscillations in Binary Stars", Granada, Spain 2004 (ASP Conf. Ser. 333, Eds. A. Claret, A. Giménez \& J.-P. Zahn); "The Astrophysics of Cataclysmic Variables and Related Objects", Strasbourg, France 2004 (ASP Conf. Ser. 330, Eds. J.M. Hameury \& J.P. Lasota); "Light Time Effect in Astrophysics: Causes and Cures of the O-C Diagram", Brussels 2004 (ASP Conf. Ser. 335, Ed. C. Sterken); "Close Binaries in the 21st Century: New Opportunities and Challenges", Syros, Greece 2005 (to be published in Ap\&SS); "A Life with Stars" Amsterdam, August 2005; "PhD Conference on Astrophysics of Variable Stars", Pécs, Hungary, September 2005 (to be published in ASP Conf. Ser.).

With respect to books, a new contribution is soon to be published in our field: "Evolutionary Processes in Binary and Multiple Stars" by Peter Eggleton (2006, Cambridge Astrophysics No. 40, ISBN-10: 0521855578).

\section{Eclipsing and Massive Binaries}

There has been remarkable progress in spectroscopic and light curve investigations of close binaries over the last three years, and observers can find summaries on-line of spectroscopic orbits (by Pourbaix et al. at http://sb9.astro.ulb.ac.be), light curves (by Bradstreet at al. at http://ebola.eastern.edu), and times of minimum light (by Kreiner at http: //www . as.wsp.krakow.pl/ephem/). Especially noteworthy are the studies of the absolute parameters for the intermediate-mass eccentric eclipsing binary MU Cas (Lacy et al. 2004, AJ 128, 1840), the high Galactic latitude eclipsing binary IT Lib that contains a pair of B-type stars ejected from the Galactic plane (Martin 2003, PASP 115, 49), the Pleiades cluster member HD 23642 (Munari et al. 2004, A\&A 418, 31), and 
50 SMC OB-type eclipsing binary systems studied by Harries et al. (2003, MNRAS 339, $157)$ and Hilditch et al. (2005, MNRAS 357,304) to determine the distance to the SMC.

Extremely large masses $\left(\approx 80 M_{\odot}\right)$ were derived for the binary components of WR 20a (Bonanos et al. 2004, ApJ 611, L33; Rauw et al. 2004, A\&A 420, L9). Rauw et al. (2005, A\&A 432, 985) suggest that the almost identical WN 6ha or O3 If*/WN 6ha components are core hydrogen burning stars in a pre-LBV stage with $\mathrm{N}$ strongly enhanced and $\mathrm{C}$ depleted in their atmospheres. Niemela \& Gamen (2005, MNRAS 356, 974) found that the brightest component of HD 150136 belongs to the spectral type O3.5 If* while the secondary is probably of type $\mathrm{O} 6$ and that there is a third star in the system. The tendency of O-type stars to be bound together in close multiple systems is also demonstrated in a new contribution of the X-Mega collaboration (Naze et al. 2005, MNRAS 359, 688) who shows that HD 93161A is a spectroscopic binary consisting of 2 O-type stars, while HD 93161B also displays radial velocity variations. Ostrov \& Lapasset (2003, MNRAS $338,1410)$ derive individual masses of 48.3 and $31.4 M_{\odot}$ for the O4f + O6 V LMC binary $\mathrm{Sk}-67^{\circ} 105$. These values are in contrast to those derived for the O4 IIIf $+\mathrm{O} 6 \mathrm{~V}$ binary Hodge 53-47 in the SMC which has individual masses of "only" 26 and $16 M_{\odot}$ (Morrell et al. 2003, MNRAS 341, 583), suggesting that a large mass range is possible for spectroscopically similar objects, according to each system's evolutionary status and mass transfer history.

Physical interactions can occur in fully detached massive binaries through collisions of their stellar winds. The wind-wind interaction zone often takes the form of a bow shock between the stars that is defined by the momentum balance of the winds. The properties of the shock region are particularly well probed through observations of the orbital phase variations in the wind lines formed in far-UV spectrum (St-Louis et al. 2005, ApJ 628, 953). The densities in the collision zone may be high enough to promote grain formation in WR+O binaries at times near periastron (Marchenko et al. 2003, ApJ 596, 1295). The colliding winds phenomenon may help explain the 5.5 year cycle of spectroscopic variations and X-ray eclipses observed in Eta Carina (Stahl et al. 2005, A\&A 435, 303; Corcoran 2005, AJ 129, 2018; Falceta-Gonçalves et al. 2005, MNRAS 357, 895).

Mass transfer in semi-detached binaries leads to the Algol-phase in which the lower mass donor star appears more evolved than the companion mass gainer. Algol systems are generally found among intermediate-mass stars but recent work has also led to the discovery of similar systems among high-mass stars (Penny et al. 2002, ApJ 575, 1050) and low-mass stars (Eggleton \& Kiseleva-Eggleton 2002, ApJ 575, 461; Torres et al. 2003, AJ 125, 3237; Mader et al. 2005, AJ 130, 234). Mass transfer in some circumstances will lead to the spin-up of the mass gainer (Barai et al. 2004, ApJ 608, 989), and when the gainer star reaches critical rotation it may no longer accrete gas from the companion (Petrovic et al. 2005, A\&A 435, 1013). The excess gas may be ejected through an outflowing disk that obscures the photosphere of the mass gainer, which may explain the near invisibility of the massive components of the W Serpentis class binaries such as $\beta$ Lyr (Harmanec 2002, AN 323, 87), the LMC star R 81 (Tubbesing et al. 2002, A\&A 389, 931), and RY Scuti (Sahade et al. 2002, RMxAA 38, 259). The remnants of the outflow in RY Scuti appear as a huge double-torus in high resolution images obtained with HST (Smith et al. 2002, ApJ 578, 464). Disks may become even larger and brighter in more advanced evolutionary stages when the mass gainer is a neutron star or black hole. The flux from the super-Eddington disk in the famous binary SS 433 is so dominant that it has frustrated past efforts to find the spectral features of the mass donor, but recent observations by Hillwig et al. (2004, ApJ 615, 422), made at a time optimal for detection of the companion, indicate that the mass donor is an A-supergiant. 


\section{Contact Binaries}

On the surface, it may seem that the field of contact binaries is in perfect situation: Many papers appear every year and many binaries are observed for light curves and timing of minima. From time to time, semi-revelations appear on unusually strong contact or, more significantly, an extremely small mass ratio (now around 0.066 for SX $\mathrm{Crv}$ ). However, the seemingly vigorous observational activity has not resulted in an improvement of our understanding of contact binaries. What we all agree is that these are main-sequence binaries with minimal angular momentum and with a special structure making it possible for components of very disparate mass to have identical effective temperature, probably thanks to some extremely vigorous energy exchange, hidden from our view inside a common envelope. Thus, we all believe in Lucy's model of 1968, a real turning point for the field whose importance still remains to be matched. Beyond this, things become difficult and blurred: We have still no idea how the energy is exchanged and how the energy transfer relates to the radial/convective dichotomy of the common envelopes (apparently it does not). We have no idea about the internal mass transfer and whether magnetic fields are important or just marginal in the whole picture. We do not know how contact binaries originate and we have sketchy ideas of what will happen to them as they evolve. Some general answers were given in the 1980's in a number of theoretical papers but very little has been added later. A glimmer of hope comes from the very new idea of horizontal flow through the differential-rotation energy advection (Yakut \& Eggleton, 2005, ApJ 629, 1055), but this must be developed more fully.

Even the spatial density numbers are unreliable, but one can expect that sky surveys will soon improve the statistics. At present, the secure data on the spatial density (Rucinski 2002, PASP 114, 1124) are from the Hipparcos survey down to only $\mathrm{V}=$ $7.5 \mathrm{mag}$ (this survey is, paradoxically, the only reasonable source of photometric calibrated data as most observers still use instrumental systems). The density appears to be high, at about 1/500 among FGK dwarfs (as Duerbeck suggested in 1984), but not as high as in the OGLE-I data of 1/130; either the inner-disk density is high close to the Bulge or the OGLE data are biased by blending (probably both). The sharp short-period cut-off has been shifted a bit from CC Com at $0.2207 \mathrm{~d}$ to $0.2155 \mathrm{~d}$ by the discovery of a system in 47 Tuc (Waldrake et al. 2004, AJ 128, 236). As expected for a Pop.II contact binary (Rucinski 2000, AJ 120, 319) it is more compact than for Pop.I.

In contrast to the flood of photometric data, spectroscopy of contact binaries lags much behind. The only current survey of bright ( $<10 \mathrm{mag})$ short-period binaries is being conducted in the Northern hemisphere (Rucinski et al. 2005, AJ 130, 767) which has given 76 radial velocity orbits of contact binaries. There is no similar survey in the South. The only radial velocity data for some Southern systems were published by Hilditch and collaborators in the 1980's. One can argue that light curves without spectroscopic support are rather useless. The Broadening Function (BF) technique gives 1-D views of contact binaries in RV space at various phases. Modeling of BF's in the 2-D (RV, time) domain should lift many degeneracies of light curve fits, but it still remains to be fully developed. The BF should show us spots and this is important as pointed out by Barnes et al. (2004, MNRAS 348, 1321) showing that a failure to model spots may lead to incorrect orbital parameters.

Timing of eclipses, while potentially useful, does not give a clear picture of what is going on. Gradual period changes do happen with characteristic times scales of $10^{5}$ to $10^{7}$ years, but these scales usually change even for one object. The period changes may come from some internal structural re-arrangements, or from third-body perturbations. The latter are surprisingly common. A very preliminary look at the situation of companions, 
disregarding discovery biases and severe limitations on classes of companions (Pribulla \& Rucinski 2005) gives the lower limit to the apparent triple-star frequency of about $50 \%$ to $65 \%$, a number not inconsistent with all contact binaries occurring in triple systems. Thus, what appeared to be a curiosity may be actually a clue to understanding their formation.

\section{Cataclysmic Variables}

A number of important theoretical and observational developments have taken place since the General Assembly in Sydney. In a series of papers by Townsley \& Bildsten (2003, ApJ, 596 227; 2004, ApJ 600, 390), they show that measured values of $T_{\text {eff }}$ yield long term accretion rates. The discovery of non-radial oscillations in the cool white dwarf (WD) primaries of short period Cataclysmic Variables (CV), like GW Lib (Szkody et al. 2002, ApJ 575, L79; van Zyl et al. 2004, MNRAS 350, 307), strongly suggests a still-unidentified mechanism driving non-radial pulsations. However, the boundaries of an instability strip have not yet been established and the oscillations are not seen in CV primaries having the same $T_{\text {eff }}$ as the pulsators. Woudt et al. (2004, ASP Conf. Ser. 330, 325) predict that $\sim 32$ out of 400 CVs discovered by the Sloan Digital Sky Survey will turn out to be ZZ Ceti-like pulsators. In the past 3 years, 7 additional ZZ Cet stars in CVs have been identified bringing the total to 8 (see http://quetzal.csc.warwick.ac.uk/phsaap/pulsators.html). These objects may well serve as exciting new candidates for asteroseismological studies where questions such as how accretion affects the internal structure of white dwarfs and their rotation rates can be explored. Theoretical explorations of this newly discovered pulsational phenomenon are already underway (Arras et al. 2005, ASP Conf. Ser. 330, 251; Townsley et al. 2004, ApJ 608, 105).

New attempts to solve for the boundary layer between the accretion disk and the accreting star, since Kippenhahn \& Thomas (1978, A\&A 63, 265) and Kutter \& Sparks (1987, ApJ 321, 386) probe the physics of how the material actually joins the WD. From the work of Inogamov \& Sunyaev (1999, Astr.Lett. 25, 269), who considered the spread of matter over a neutron star surface during disk accretion, Piro \& Bildsten (2005, ASP Conf. Ser. 330, 197; 2004, ApJ, 616, 155; 2004, ApJ, 610, 977) have extended the semianalytic numerical treatment to the white dwarf case and found that the spreading of the material is rapid during outburst, an accretion belt forms but is undetectable during quiescence, and the spin down of the belt is rapid. Moreover, the controlling viscosity is due to turbulence. However, the first 2-D and 3-D hydrodynamic simulations of this process are currently in progress (see Fisker \& Balsara 2005, AAS 206, 43.06).

For magnetic systems, an XMM snapshot survey of about 40 polars by Ramsey \& Cropper (2004, ASP Conf. Ser. 315, 106) suggests that the vast majority of polars show no 'soft X-ray excess'. Their XMM studies also show that most polars spend most of their time in low accretion states. Studies of Low Accretion Rate Polars (LARP) show that these may be pre-magnetic CVs containing the oldest, coolest white dwarfs that are accreting only via a wind (Schmidt et al. 2005, ApJ 630, L173). A different evolution is also signified by different abundances of secondaries in magnetic versus disk systems (Harrison et al. 2005, AJ 129, 2400).

The July 2001 super-outburst of WZ Sge (ten years earlier than expected) has now been reasonably well characterized in a wealth of papers on the optical behavior (Patterson et al. 2002, PASP 115, 1308), the WD properties (Sion et al. 2003, ApJ 592, 1137; Long et al. 2004, ApJ 602, 948; Long et al. 2003, ApJ 591, 472), the physics of the WD cooling (Godon et al. 2004, ApJ 602, 336), the discovery of 15 s oscillations (Knigge et al. 2002, ApJ 580, L151), and the detection of the 28 s oscillations which cannot be due to 
non-radial g-modes (Welsh, et al. 2003, ApJ 599, 509). But puzzles still remain. The WD mass remains elusive although it appears to be $\sim 0.9 M_{\odot}$ based upon Steeghs et al. (2001, ApJ 562, L145) radial velocity mass of the secondary during the outburst. Using its well-determined parallax (43 pc) we know the outburst energy, the accretion rate during outburst, the amount of heating by the outburst and the rate of cooling of the WD by compressional heating. The persistence of UV oscillations despite the WD cooling by several thousand degrees supports a rotational explanation as the clock for the $28 \mathrm{~s}$ oscillations but the rotation rate of the WD remains unknown as well as the formation region of FUV absorption lines.

\section{Very low-mass stars in close binary systems}

The observation and study of very low-mass stars is a field in rapid development. This is mostly a consequence of the increasing number of deep photometric surveys and the advent of powerful instrumentation able to obtain spectroscopy of these intrinsically faint objects. But also renewed interest arises from one of the "hot topics" of this past decade: exoplanets. Very low mass brown dwarfs and giant planets share many physical characteristics and their study and modeling are often intimately related. With the huge observational effort being focused on exoplanets and brown dwarfs, often some interesting results on low-mass stars occur as "by-products".

Very few M-type stars were known in close binary systems until recently. In these past three years, several new discoveries have been reported and studied. From these, a discrepancy between the stellar radii predicted by models and the measurements from observations has become increasingly evident. In the cases of CU Cnc (Ribas 2003, A\&A 398, 239) and GU Boo (López-Morales \& Ribas 2005, ApJ), where the masses and radii of the components could be measured to an accuracy of a few percent, the stars were found to be some $5-10 \%$ larger than model predictions. This is in agreement with results from a previous analysis of a third M-type eclipsing binary, YY Gem. The discrepancy is not so compelling in the case of OGLE BW3 V38 (Maceroni \& Montalbán 2004, A\&A $426,577)$ because of the larger observational uncertainties. The system TrES-Her0-07621 (Creevey et al. 2005, ApJ 625, L127) has components with radii that seem to agree better with theoretical predictions but the observational error bars are still too large to draw any conclusion. Efforts in the coming years should be undertaken to bring observation and theory to an agreement.

Large-scale surveys have also contributed to increase the number of close binaries with M-type components. Follow-up of OGLE planetary transit candidates has resulted in several systems with $\mathrm{F}-\mathrm{G}$ primaries and M-type secondaries (Bouchy et al. 2005, A\&A 431, 1105; Pont et al. 2005, A\&A 438, 1123). The fundamental properties of these stars (masses and radii), however, could only be determined by making certain modeldependent assumptions since the light curves display one eclipse (transit of the M-type component) and the spectrum is single lined. The same follow-up observations have also uncovered a transiting M-dwarf star in the system OGLE-TR-122 that has the same radius as a giant planet (Pont et al. 2005, A\&A 433, L21).

Besides systems with main sequence components, a number of close binaries with M-type stars and evolved companions have been reported. The systems EC13471-1258 (O'Donoghue et al. 2003, MNRAS 345, 506) and RXJ2130.6+4710 (Maxted et al. 2004, MNRAS 355, 1143) have eclipsing M dwarf/white dwarf components, while HS 2333+3927 (Heber et al. 2004, A\&A 420, 251) is composed of a subdwarf B star with an M-dwarf companion. Some of these systems may be the progenitors of cataclysmic variables, which makes them worthy of detailed study. 
Close binary systems with brown dwarf companions are the natural additional step down in mass. Theoretical estimations (Pinfield et al. 2005, PASP 117, 173) indicate that upcoming transit experiments should discover eclipsing brown dwarfs by the hundreds. In the meantime, some brown dwarf binaries have been identified and observed, such as GJ 569 Bab, which is the first brown dwarf binary for which dynamical masses have been determined (Zapatero Osorio et al. 2004, ApJ 615, 958). There has also been the first report of a pair of eclipsing brown dwarfs in the Orion star forming region (Gómez Maqueo Chew et al. 2005, in AAS Meeting 206). Brown dwarfs have been suggested as companions of white dwarfs (Schmidt et al. 2005, ApJ 630, L173) and as secondaries in short-period cataclysmic variables (Mennickent et al. 2004, MNRAS 347, 1180).

Low-mass star research has also been very active in performing direct detections using adaptive optics and/or interferometry. Many have been the publications reporting new discoveries and orbital solutions of binary systems with very low mass companions. Although these systems would have traditionally been referred to as visual binaries, the increasing instrumental capabilities are bridging the gap with the "classical" close binaries. The strong interest in this field promises new exciting results and discoveries during the coming years.

\section{Surveys}

Surveys are the subject of a lot of attention in recent years and have proven to be very rewarding for the study of close binaries. Robotic telescopes and wide-field photometric surveys are rapidly expanding. Many new discoveries have allowed to attempt statistical studies previously not possible. A review of the most recent results is given in the different sections of this report but in the case of cataclysmic variables some additional comments are necessary.

The Sloan Digital Sky Survey now contains photometry of 180 million objects over 6670 square degrees and spectroscopy of over 600,000 objects, including many close binaries. The data through 2004 has revealed 128 new CVs (Szkody et al. 2005, AJ 129, 238), 5 new AM CVn (including one eclipsing system; Anderson et al. 2005, AJ) and over 700 non-interacting WD plus M-dwarf pairs (Silvestri et al. 2005, AJ). The majority of the CVs have low accretion rates, some so low that they are accreting only via a wind. Several dozen show the underlying stars, 4 of which have been found to contain pulsating white dwarfs. This is in contrast to the follow-up work from the 50 new CVs found in the Hamburg survey (Gänsicke et al. 2005), which is revealing higher accretion rate systems including several new SW Sex and Intermediate Polars. Chandra and XMM surveys of globular clusters are showing that many CV candidates exist within clusters. Follow-up observations with HST and optical are being used to identify the specific types of objects that are producing the X-rays.

A new development of importance for close binary stars is also related to Virtual Observatories. The combination of space- and ground-based observations, including large surveys databases, is clearly becoming an excellent new tool. Among the techniques being developed during the past triennium, required in virtual observatories for the study of close binaries, is the automatic classification of individual systems based on neural networks.

\section{New missions}

Several comments have been given already in the previous sections related to the current missions in orbit, mainly XMM and Chandra in the X-ray domain, and HST in 
the optical. New unforeseen data was provided by Integral in gamma-rays allowing to discover highly obscured X-ray binaries as well as the study of the high-energy emission of compact systems. On the other hand Spitzer is providing important information on stellar formation, including binaries, and GALEX is allowing the ultraviolet extension of detailed studies of close binaries.

Furthermore MOST, in the optical, has shown the capability of space telescopes for accurate photometry and high-precision monitoring of the photometric behavior of stars leading to asteroseismology. In the coming triennium, two new space missions are going to be the drivers for an improved study of close binaries using wide-field high-precision photometric monitoring: COROT to be launched in 2006 and Kepler planned for two years later.

\section{Working Group on Accretion Physics in Interacting Binaries}

The working group has continued to summarize its results for dissemination in the Interacting Binaries Electronic Newsletter, which is accessed through the C42 web site. A session on accretion across several disciplines is planned for Prague and many members are planning to attend the $4-$ month program at the Kavli Institute for Theoretical Physics on Accretion and Explosion: The Astrophysics of Degenerate Stars.

\section{Working Group on Variable and Binary Stars in Galaxies}

At the time of writing of this report, a new Working Group (WG) with the name "Variable and Binary Stars in Galaxies", sponsored by Division V, is being established. This WG is born with the main goal of promoting research in extra-galactic variable and binary stars. The flow of data from the micro-lensing surveys has been so phenomenal that a large number of investigators are now working in this field. The recent IAU Colloquium 193 "Variable Stars in the Local Group" and Joint Discussion 13 on "Extragalactic Binaries" held in 2003 in New Zealand and Australia, respectively, are a clear reflection of the increasing interest. A WG on both variables and binaries will provide an excellent forum for discussion and exchange among the members of this rapidly developing community. The activities may include the creation of a periodic newsletter and the WG could help to organize and coordinate meetings on the subject. It should also be emphasized that data mining in large catalogs is an excellent way to do cutting-edge science for researchers in countries with limited resources and the existence of a WG will represent an opportunity for them to establish collaborations. Finally, the new WG could coordinate the efforts to create a homogeneous database of extra-galactic variable stars and perhaps set some naming standards, since the current situation is rather chaotic. 